\title{
Two-Frequency Radiative Transfer and Asymptotic Solution
}

\author{
Albert C. Fannjiang 1 \\ Department of Mathematics, University of California, Davis, CA 95616-8633 \\ fannjiang@math.ucdavis.edu
}

Two-frequency radiative transfer (2f-RT) theory is developed for classical waves in random media. Depending on the ratio of the wavelength to the scale of medium fluctuation 2f-RT equation is either a Boltzmann-like integral equation with a complex-valued kernel or a Fokker-Planck-like differential equation with complex-valued coefficients in the phase space. The $2 \mathrm{f}-\mathrm{RT}$ equation is used to estimate three physical parameters: the spatial spread, the coherence length and the coherence bandwidth (Thouless frequency). A closed form solution is given for the boundary layer behavior of geometrical radiative transfer and shows highly nontrivial dependence of mutual coherence on the spatial displacement and frequency difference. It is shown that the paraxial form of $2 \mathrm{f}-\mathrm{RT}$ arises naturally in anisotropic media which fluctuate slowly in the longitudinal direction. (c) 2013 Optical Society of America

OCIS codes: 030.5620, 290.4210

\section{Introduction}

Let $U_{j}, j=1,2$, be the random, scalar wave field of wavenumber $k_{j}, j=1,2$, The mutual coherence function and its cross-spectral version, known as the two-frequency mutual coherence function, defined by

$$
\Gamma_{12}(\mathbf{x}, \mathbf{y})=\left\langle U_{1}\left(\frac{\mathbf{x}}{k_{1}}+\frac{\mathbf{y}}{2 k_{1}}\right) U_{2}^{*}\left(\frac{\mathbf{x}}{k_{2}}-\frac{\mathbf{y}}{2 k_{2}}\right)\right\rangle,
$$

where $\langle\cdot\rangle$ stands for the ensemble averaging, is the central quantity of optical coherence theory, from which the two-space, two-time correlation function can be obtained via Fourier transform in frequency, and therefore plays a fundamental role in analyzing propagation of random pulses. ${ }^{3,4,17,18,21}$ The motivation for the scaling factors in (11) will be given below, cf. (3). 
In this paper, we set out to analyze the two-frequency mutual coherence as function of the spatial displacement and frequency difference for classical waves in multiply scattering media. This problem has been extensively studied in the physics literature $\left(\mathrm{see}^{2,17,23,26}\right.$ and references therein). Here we derive from the multscale expansion (MSE) the two-frequency version of the radiative transfer equation which is then used to estimate qualitatively the three physical parameters: the spatial and spatial frequency spreads, and the coherence bandwidth, also known as the Thouless frequency in condensed matter physics. Moreover, we show that the boundary layer behavior of the two-frequency radiative transfer (2f-RT) equation is analytically solvable in geometrical optics. The closed form solution (43) provides detailed information of the two-frequency mutual coherence beyond the current physical picture $^{23,24,26}$ (see the discussion about (44)).

To this end, we introduce the two-frequency Wigner distribution whose ensemble average is equivalent to the two-frequency mutual coherence and is a natural extension of the standard Wigner distribution widely used in optics. ${ }^{6,14}$ A different version of two-frequency Wigner distribution for parabolic waves was introduced earlier ${ }^{8}$ and with it the corresponding radiative transfer equation has been derived with full mathematical rigor. ${ }^{11,12}$ In the case of anisotropic media fluctuating slowly in the longitudinal direction the $2 \mathrm{f}-\mathrm{RT}$ equation developed here reduces to that of the paraxial waves in similar media which lends support to the validity of MSE. The other regime where the two frequency radiative transfer equation has been obtained with full mathematical rigor is geometrical optics. ${ }^{13}$

The main difference between the 2f-RT and the standard theory is that the former retains the wave nature of the process and is not just about energy transport. Hence the governing equation can not be derived simply based on the energy conservation law.

\section{Two-frequency Wigner distribution}

Let $U_{j}, j=1,2$ be governed by the reduced wave equation

$$
\Delta U_{j}(\mathbf{r})+k_{j}^{2}\left(\nu_{j}+V_{j}(\mathbf{r})\right) U_{j}(\mathbf{r})=f_{j}(\mathbf{r}), \quad \mathbf{r} \in \mathbb{R}^{3}, \quad j=1,2
$$

where $\nu_{j}$ and $V_{j}$ are respectively the mean and fluctuation of the refractive index associated with the wavenumber $k_{j}$ and are in general complex-valued. The source terms $f_{j}$ may result from the initial data or the external sources. Here and below the vacuum phase speed is set to be unity. To solve (2) one needs also some boundary condition which is assumed to be vanishing at the far field.

We define the two-frequency Wigner distribution as

$$
W(\mathbf{x}, \mathbf{p})=\frac{1}{(2 \pi)^{3}} \int e^{-i \mathbf{p} \cdot \mathbf{y}} U_{1}\left(\frac{\mathbf{x}}{k_{1}}+\frac{\mathbf{y}}{2 k_{1}}\right) U_{2}^{*}\left(\frac{\mathbf{x}}{k_{2}}-\frac{\mathbf{y}}{2 k_{2}}\right) d \mathbf{y} .
$$

In view of the definition, we see that both $\mathbf{x}$ and $\mathbf{p}$ are dimensionless. Here the choice of the

scaling factors is crucial; namely, the spatial dependence of the wave field should be measured 
w.r.t. the probing wavelength. The benefit is that this choice leads to a closed form equation for $W$. It is easy to see that the ensemble average $\langle W\rangle$ is just the (partial) Fourier transform of the mutual coherence function (11). The two-frequency Wigner distribution defined here has a different scaling factor from the one introduced for the parabolic waves. ${ }^{8}$

The purpose of introducing the two-frequency Wigner distribution is to develop a twofrequency theory in analogy to the well studied standard theory of radiative transfer. Although the definition (3) requires the domain to be $\mathbb{R}^{3}$, the governing radiative transfer equation, once obtained, can be (inverse) Fourier transformed back to get the governing equation for the two-point function $U_{1}\left(\mathbf{r}_{1}\right) U_{2}^{*}\left(\mathbf{r}_{2}\right)$ or $\Gamma_{12}$ as their boundary conditions are usually easier to describe (cf. eq. (42)).

The Wigner distribution has the following easy-to-check properties:

$$
\begin{aligned}
\int|W|^{2}(\mathbf{x}, \mathbf{p}) d \mathbf{x} d \mathbf{p} & =\left(\frac{\sqrt{k_{1} k_{2}}}{2 \pi}\right)^{3} \int\left|U_{1}\right|^{2}(\mathbf{x}) d \mathbf{x} \int\left|U_{2}\right|^{2}(\mathbf{x}) d \mathbf{x} \\
\int W(\mathbf{x}, \mathbf{p}) e^{i \mathbf{p} \cdot \mathbf{y}} d \mathbf{p} & =U_{1}\left(\frac{\mathbf{x}}{k_{1}}+\frac{\mathbf{y}}{2 k_{1}}\right) U_{2}^{*}\left(\frac{\mathbf{x}}{k_{2}}-\frac{\mathbf{y}}{2 k_{2}}\right) \\
\int W(\mathbf{x}, \mathbf{p}) e^{-i \mathbf{x} \cdot \mathbf{q}} d \mathbf{x} & =\left(\pi^{2} k_{1} k_{2}\right)^{3} \widehat{U}_{1}\left(\frac{k_{1} \mathbf{p}}{4}+\frac{k_{1} \mathbf{q}}{2}\right) \widehat{U}_{2}^{*}\left(\frac{k_{2} \mathbf{p}}{4}-\frac{k_{2} \mathbf{q}}{2}\right),
\end{aligned}
$$

where $\widehat{r}$ stands for the Fourier transform, and hence contains all the information in the two-point two-frequency function. In particular,

$$
\int \mathbf{p} W(\mathbf{x}, \mathbf{p}) d \mathbf{p}=-i\left[\frac{1}{2 k_{1}} \nabla U_{1}\left(\frac{\mathbf{x}}{k_{1}}\right) U_{2}^{*}\left(\frac{\mathbf{x}}{k_{2}}\right)-\frac{1}{2 k_{2}} U_{1}\left(\frac{\mathbf{x}}{k_{1}}\right) \nabla U_{2}^{*}\left(\frac{\mathbf{x}}{k_{2}}\right)\right]
$$

which, in the case of $k_{1}=k_{2}$, is proportional to the energy flux density.

We now derive the equation for the two-frequency Wigner distribution. After taking the derivative $\mathbf{p} \cdot \nabla$ and some calculation we have

$$
\begin{aligned}
\mathbf{p} \cdot \nabla W= & \frac{i}{2(2 \pi)^{3}} \int e^{-i \mathbf{p} \cdot \mathbf{y}} U_{1}\left(\frac{\mathbf{x}}{k_{1}}+\frac{\mathbf{y}}{2 k_{1}}\right) U_{2}^{*}\left(\frac{\mathbf{x}}{k_{2}}-\frac{\mathbf{y}}{2 k_{2}}\right) V_{1}\left(\frac{\mathbf{x}}{k_{1}}+\frac{\mathbf{y}}{2 k_{1}}\right) d \mathbf{y} \\
& -\frac{i}{2(2 \pi)^{3}} \int e^{-i \mathbf{p} \cdot \mathbf{y}} U_{1}\left(\frac{\mathbf{x}}{k_{1}}+\frac{\mathbf{y}}{2 k_{1}}\right) U_{2}^{*}\left(\frac{\mathbf{x}}{k_{2}}-\frac{\mathbf{y}}{2 k_{2}}\right) V_{2}^{*}\left(\frac{\mathbf{x}}{k_{2}}-\frac{\mathbf{y}}{2 k_{2}}\right) d \mathbf{y} \\
& +\frac{i}{2}\left(\nu_{1}-\nu_{2}^{*}\right) W+F
\end{aligned}
$$

where the function $F$ depends linearly on $U_{j}$ and $f_{j}$ :

$$
\begin{aligned}
F= & -\frac{i}{2(2 \pi)^{3}} \int e^{-i \mathbf{p} \cdot \mathbf{y}} f_{1}\left(\frac{\mathbf{x}}{k_{1}}+\frac{\mathbf{y}}{2 k_{1}}\right) U_{2}^{*}\left(\frac{\mathbf{x}}{k_{2}}-\frac{\mathbf{y}}{2 k_{2}}\right) d \mathbf{y} \\
& +\frac{i}{2(2 \pi)^{3}} \int e^{-i \mathbf{p} \cdot \mathbf{y}} U_{1}\left(\frac{\mathbf{x}}{k_{1}}+\frac{\mathbf{y}}{2 k_{1}}\right) f_{2}^{*}\left(\frac{\mathbf{x}}{k_{2}}-\frac{\mathbf{y}}{2 k_{2}}\right) d \mathbf{y} .
\end{aligned}
$$

Substituting the spectral representation of $V_{j}$

$$
V_{j}(\mathbf{x})=\int e^{i \mathbf{q} \cdot \mathbf{x}} \hat{V}_{j}(d \mathbf{q})
$$


in the expression and using the definition of $W$ we then obtain the exact equation

$$
\begin{aligned}
\mathbf{p} & \cdot \nabla W-\frac{i}{2}\left(\nu_{1}-\nu_{2}^{*}\right) W-F \\
= & \frac{i}{2} \int \hat{V}_{1}(d \mathbf{q}) e^{i \mathbf{q} \cdot \mathbf{x} / k_{1}} W\left(\mathbf{x}, \mathbf{p}-\frac{\mathbf{q}}{2 k_{1}}\right)-\frac{i}{2} \int \hat{V}_{2}^{*}(d \mathbf{q}) e^{-i \mathbf{q} \cdot \mathbf{x} / k_{2}} W\left(\mathbf{x}, \mathbf{p}-\frac{\mathbf{q}}{2 k_{2}}\right) .
\end{aligned}
$$

Here and below $\hat{V}_{2}^{*}$ is the complex-conjugate of the Fourier spectral measure $\hat{V}_{2}$. The full derivation of (9) is given in Appendix A.

Let us pause to compare the classical wave with the quantum wave function in the context of two-frequency formulation. The quantum wave functions $\Psi_{j}$ at two different frequencies $\omega_{1}, \omega_{2}$ satisfy the stationary Schrödigner equaiton

$$
\frac{\hbar^{2}}{2} \Delta \Psi_{j}+\left(\nu_{j}+V_{j}(\mathbf{x})\right) \Psi_{j}=-\omega_{j} \hbar \Psi_{j}+f_{j}, \quad j=1,2
$$

where $\nu_{j}+V_{j}$ are hypothetical, energy-dependent real-valued potentials. Here the source terms $f_{j}$ equal the initial data $f$ of the time dependent problem. Usually in the quantum mechanical context, the potential function does not explicitly depend on the energy level (i.e. dispersionless).

The natural definition of the two-frequency Wigner distribution for the quantum wave functions is

$$
W(\mathbf{x}, \mathbf{p})=\frac{1}{(2 \pi)^{3}} \int e^{-i \mathbf{p} \cdot \mathbf{y}} \Psi_{1}\left(\mathbf{x}+\frac{\hbar \mathbf{y}}{2}\right) \Psi_{2}^{*}\left(\mathbf{x}-\frac{\hbar \mathbf{y}}{2}\right) d \mathbf{y}
$$

which satisfies the Wigner-Moyal equation

$$
\begin{aligned}
\mathbf{p} & \cdot \nabla W+i\left(\omega_{2}-\omega_{1}\right) W+\frac{i}{\hbar}\left(\nu_{2}^{*}-\nu_{1}\right) W \\
= & \frac{i}{\hbar} \int \hat{V}_{1}(d \mathbf{q}) e^{i \mathbf{q} \cdot \mathbf{x}} W\left(\mathbf{x}, \mathbf{p}-\frac{\hbar \mathbf{q}}{2}\right)-\frac{i}{\hbar} \int \hat{V}_{2}^{*}(d \mathbf{q}) e^{-i \mathbf{q} \cdot \mathbf{x}} W\left(\mathbf{x}, \mathbf{p}-\frac{\hbar \mathbf{q}}{2}\right)+F
\end{aligned}
$$

where $F$ has a similar expression to (7). The main difference between the quantum and classical waves in the Wigner formulation is that the derivation of a closed-form equation does not require rescaling each energy component w.r.t. its de Broglie wavelength. The implication in radiative transfer will be further discussed (see the remark following eq. (27)).

\section{Two-frequency radiative transfer scaling}

We assume that $V_{j}(\mathbf{x}), j=1,2$ are real-valued, centered, random stationary (i.e. statistically homogeneous) ergodic field admitting the spectral representation (8) with the spectral measures $\hat{V}_{j}(d \mathbf{p}), j=1,2$ such that

$$
\left\langle\hat{V}_{j}(d \mathbf{p}) \hat{V}_{j}^{*}(d \mathbf{q})\right\rangle=\delta(\mathbf{p}-\mathbf{q}) \Phi_{j}(\mathbf{p}) d \mathbf{p} d \mathbf{q}
$$


where $\Phi_{j}$ are the (nonnegative-valued) power spectral densities of the random fields $V_{j}, j=$ 1,2 . The above $\delta$ function is a consequence of the statistical homogeneity of the random field $V_{j}$. As $V_{j}, j=1,2$ are real-valued, $\hat{V}_{j}^{*}(d \mathbf{p})=\hat{V}_{j}(-d \mathbf{p})$ and hence the power spectral densities $\Phi_{j}(\mathbf{p})$ satisfy the symmetry property $\Phi_{j}(\mathbf{p})=\Phi_{j}(-\mathbf{p}), \forall \mathbf{p}$.

We will also need the cross-frequency correlation and we postulate the existence of the cross-frequency spectrum $\Phi_{12}$ such that

$$
\left\langle\hat{V}_{1}(d \mathbf{p}) \hat{V}_{2}^{*}(d \mathbf{q})\right\rangle=\delta(\mathbf{p}-\mathbf{q}) \Phi_{12}(\mathbf{p}) d \mathbf{p} d \mathbf{q}
$$

Here $\Phi_{12}$ needs not be real-valued.

An important regime of multiple scattering of classical waves takes place when the scale of medium fluctuation is much smaller than the propagation distance but is comparable or much larger than the wavelength. ${ }^{17,19}$ Radiative transfer regime can be characterized by the scaling limit which replaces $\nu_{j}+V_{j}$ in eq. (2) with

$$
\frac{1}{\theta^{2} \varepsilon^{2}}\left(\nu_{j}+\sqrt{\varepsilon} V_{j}\left(\frac{\mathbf{r}}{\varepsilon}\right)\right), \quad \theta>0, \quad \varepsilon \ll 1
$$

where $\varepsilon$ is the ratio of the scale of medium fluctuation to the $O(1)$ propagation distance and $\theta$ the ratio of the wavelength to the scale of medium fluctuation. Hence $\theta \varepsilon$ is the ratio of the wavelength to the propagation distance and the prefactor $(\theta \varepsilon)^{-2}$ arises from rescaling the wavenumber $k \rightarrow k /(\varepsilon \theta)$. This is so called the weak coupling (or disorder) limit in kinetic theory which prohibits the Anderson localization from happening. ${ }^{25}$ Note that the resulting medium fluctuation $\varepsilon^{-3 / 2} V_{j}(\mathbf{r} / \varepsilon)$ converges to a spatial white-noise in three dimensions.

Physically speaking the radiative transfer scaling belongs to the diffusive wave regime under the condition of a large dimensionless conductance $g=N \ell_{t} / L$, where $\ell_{t}$ is the transport mean free path, $L$ is the sample size in the direction of propagation and $N=2 \pi A / \lambda^{2}$ is the number of transverse modes, limited by the illuminated area $A$ and the wavelength of radiation $\lambda .^{2,23}$ The dimensionless conductance $g$ can be expressed as $g=k \ell_{t} /$ Fr with the inverse Fresnel number $\mathrm{Fr}=\lambda L / A$. With the scaling (13),$k \ell_{t} \sim \mathrm{Fr}^{-1} \sim \theta^{-1} \varepsilon^{-1}$ and hence $g \sim \theta^{-2} \varepsilon^{-2} \gg 1$ for any finite $\theta$ as $\varepsilon \rightarrow 0$.

Anticipating small-scale fluctuation due to (13) we modify the definition of the twofrequency Wigner distribution in the following way

$$
W(\mathbf{x}, \mathbf{p})=\frac{1}{(2 \pi)^{3}} \int e^{-i \mathbf{p} \cdot \mathbf{y}} U_{1}\left(\frac{\mathbf{x}}{k_{1}}+\frac{\theta \varepsilon \mathbf{y}}{2 k_{1}}\right) U_{2}^{*}\left(\frac{\mathbf{x}}{k_{2}}-\frac{\theta \varepsilon \mathbf{y}}{2 k_{2}}\right) d \mathbf{y}
$$

Eq. (9) now becomes

$$
\mathbf{p} \cdot \nabla W-F=\frac{i}{2 \varepsilon \theta}\left(\nu_{1}-\nu_{2}^{*}\right) W+\frac{1}{\sqrt{\varepsilon}} \mathcal{L} W
$$


where the operator $\mathcal{L}$ is defined by

$$
\mathcal{L} W(\mathbf{x}, \mathbf{p})=\frac{i}{2 \theta} \int \hat{V}_{1}(d \mathbf{q}) e^{i \frac{\mathbf{q} \cdot \mathbf{x}}{\varepsilon k_{1}}} W\left(\mathbf{x}, \mathbf{p}-\frac{\theta \mathbf{q}}{2 k_{1}}\right)-\frac{i}{2 \theta} \int \hat{V}_{2}^{*}(d \mathbf{q}) e^{-i \frac{\mathbf{q} \cdot \mathbf{x}}{\varepsilon k_{2}}} W\left(\mathbf{x}, \mathbf{p}-\frac{\theta \mathbf{q}}{2 k_{2}}\right)
$$

To capture the cross-frequency correlation in the radiative transfer regime we also need to restrict the frequency difference range

$$
\lim _{\varepsilon \rightarrow 0} k_{1}=\lim _{\varepsilon \rightarrow 0} k_{2}=k, \quad \frac{k_{2}-k_{1}}{\varepsilon \theta k}=\beta
$$

where $k, \beta>0$ are independent of $\varepsilon$ and $\theta$. Assuming the differentiability of the mean refractive index's dependence on the wavenumber we write

$$
\frac{\nu_{2}^{*}-\nu_{1}}{2 \varepsilon \theta}=\nu^{\prime}
$$

where $\nu^{\prime}$ is independent of $\varepsilon, \theta$.

\section{Multi-scale expansion (MSE)}

To derive the radiative transfer equation for the two-frequency Wigner distribution we employ $\mathrm{MSE}^{1,20}$ which begins with introducing the fast variable

$$
\tilde{\mathbf{x}}=\mathbf{x} / \varepsilon
$$

and treating $\tilde{\mathbf{x}}$ as independent from the slow variable $\mathbf{x}$. Consequently the derivative $\mathbf{p} \cdot \nabla$ consists of two terms

$$
\mathbf{p} \cdot \nabla=\mathbf{p} \cdot \nabla_{\mathbf{x}}+\varepsilon^{-1} \mathbf{p} \cdot \nabla_{\tilde{\mathbf{x}}}
$$

Then MSE posits the following asymptotic expansion:

$$
W(\mathbf{x}, \mathbf{p})=\bar{W}(\mathbf{x}, \tilde{\mathbf{x}}, \mathbf{p})+\sqrt{\varepsilon} W_{1}(\mathbf{x}, \tilde{\mathbf{x}}, \mathbf{p})+\varepsilon W_{2}(\mathbf{x}, \tilde{\mathbf{x}}, \mathbf{p})+O\left(\varepsilon^{3 / 2}\right), \quad \tilde{\mathbf{x}}=\mathbf{x} \varepsilon^{-1}
$$

whose proper sense will be explained below.

Substituting the ansatz into eq. (14) and using (17) we determine each term of (18) by equating terms of the same order of magnitude starting with the highest order $\varepsilon^{-1}$.

The $\varepsilon^{-1}$-order equation has one term:

$$
\mathbf{p} \cdot \nabla_{\tilde{\mathbf{x}}} \bar{W}=0
$$

which can be solved by setting $\bar{W}=\bar{W}(\mathbf{x}, \mathbf{p})$. Namely, to the leading order $W$ is independent of the fast variable. Since the fast variable is due to medium fluctuation, this suggests that $\bar{W}$ is deterministic. 
The next is the $\varepsilon^{-1 / 2}$-order equation:

$$
\mathbf{p} \cdot \nabla_{\tilde{\mathbf{x}}} W_{1}=\mathcal{L} \bar{W}
$$

We seek a solution that is stationary in $\tilde{\mathbf{x}}$, square-integrable in $\mathbf{p}$ and has finite second moment. The solvability condition (Fredholm alternative) is that the right hand side, $\mathcal{L} \bar{W}$, satisfies $\int \mathbb{E}\left[\Psi^{*} \mathcal{L} \bar{W}\right] d \mathbf{p}=0$ for any $\tilde{\mathbf{x}}$-stationary, square-integrable field $\Psi(\tilde{\mathbf{x}}, \mathbf{p})$ satisfying $\mathbf{p} \cdot \nabla_{\tilde{\mathbf{x}}} \Psi=0$. The solvability condition is, however, not easy to enforce. Alternatively we consider the regularized equation

$$
\varepsilon W_{1}^{\varepsilon}+\mathbf{p} \cdot \nabla_{\tilde{\mathbf{x}}} W_{1}^{\varepsilon}=\mathcal{L} \bar{W}
$$

which is always solvable for $\varepsilon>0$ and admits the solution

$$
\begin{aligned}
W_{1}^{\varepsilon}(\mathbf{x}, \tilde{\mathbf{x}}, \mathbf{p})= & \frac{i}{2 \theta} \int \hat{V}_{1}(d \mathbf{q}) \frac{e^{i \frac{\mathbf{q} \cdot \tilde{x}}{k_{1}}}}{\varepsilon+i \mathbf{q} \cdot \mathbf{p} / k_{1}} \bar{W}\left(\mathbf{x}, \mathbf{p}-\frac{\theta \mathbf{q}}{2 k_{1}}\right) \\
& -\frac{i}{2 \theta} \int \hat{V}_{2}^{*}(d \mathbf{q}) \frac{e^{-i \frac{\mathbf{q} \cdot \tilde{\mathbf{x}}}{k_{2}}}}{\varepsilon-i \mathbf{q} \cdot \mathbf{p} / k_{2}} \bar{W}\left(\mathbf{x}, \mathbf{p}-\frac{\theta \mathbf{q}}{2 k_{2}}\right) .
\end{aligned}
$$

In the jargons of asymptotic analysis, ${ }^{1} \sqrt{\varepsilon} W_{1}^{\varepsilon}$ is called the first corrector. In order to control the first corrector, we choose $\bar{W}$ such that $\mathcal{L} \bar{W}$ has zero mean. This is a necessary condition as we seek a $\tilde{\mathbf{x}}$-stationary solution and consequently $\left\langle\mathbf{p} \cdot \nabla_{\tilde{\mathbf{x}}} W_{1}\right\rangle=\mathbf{p} \cdot \nabla_{\tilde{\mathbf{x}}}\left\langle W_{1}\right\rangle=0$. Needless to say, this condition is weaker than the solvability condition stated above and is satisfied for any deterministic $\bar{W}$ since both $V_{1}$ and $V_{2}$ have zero mean.

Indeed, under the assumption of deterministic $\bar{W}$, the resulting equation will be much simplified so we impose this property on $\bar{W}$ from now on. The fact that in the limit $\bar{W}$ is deterministic can be proved rigorously in the paraxial regime. ${ }^{12}$

Finally the $O(1)$ equation is

$$
\begin{aligned}
\mathbf{p} \cdot \nabla_{\tilde{\mathbf{x}}} W_{2}(\mathbf{x}, \tilde{\mathbf{x}}, \mathbf{p})= & -\mathbf{p} \cdot \nabla_{\mathbf{x}} \bar{W}(\mathbf{x}, \mathbf{p})-i \nu^{\prime} \bar{W}+F+\frac{i}{2 \theta} \int \hat{V}_{1}(d \mathbf{q}) e^{i \frac{\mathbf{q} \cdot \tilde{\mathbf{x}}}{k_{1}}} W_{1}^{\varepsilon}\left(\mathbf{x}, \tilde{\mathbf{x}}, \mathbf{p}-\frac{\theta \mathbf{q}}{2 k_{1}}\right) \\
& -\frac{i}{2 \theta} \int \hat{V}_{2}^{*}(d \mathbf{q}) e^{-i \frac{\mathbf{q} \cdot \tilde{\mathbf{x}}}{k_{2}}} W_{1}^{\varepsilon}\left(\mathbf{x}, \tilde{\mathbf{x}}, \mathbf{p}-\frac{\theta \mathbf{q}}{2 k_{2}}\right)
\end{aligned}
$$

which can be solved with regularization as in (20) and yields the second corrector $\varepsilon W_{2}^{\varepsilon}$. Again we impose on the right hand side of (22) the weaker condition of zero mean. Using (21) in (22), taking the ensemble average and passing to the limit $\varepsilon \rightarrow 0$ we obtain the governing 
equation for $\bar{W}$ :

$$
\begin{aligned}
\mathbf{p} \cdot \nabla_{\mathbf{x}} & \bar{W}(\mathbf{x}, \mathbf{p})+i \nu^{\prime} \bar{W}-\langle F\rangle \\
= & -\frac{k_{1}^{3}}{2 \theta^{4}} \int d \mathbf{q} \Phi_{1}\left(\frac{k_{1}}{\theta}(\mathbf{p}-\mathbf{q})\right) \pi \delta\left(|\mathbf{p}|^{2}-|\mathbf{q}|^{2}\right) \bar{W}(\mathbf{x}, \mathbf{p})+\frac{i k_{1}^{3}}{2 \theta^{4}} f d \mathbf{q} \frac{\Phi_{1}\left(\frac{k_{1}}{\theta}(\mathbf{p}-\mathbf{q})\right)}{|\mathbf{p}|^{2}-|\mathbf{q}|^{2}} \bar{W}(\mathbf{x}, \mathbf{p}) \\
& -\frac{k_{2}^{3}}{2 \theta^{4}} \int d \mathbf{q} \Phi_{2}\left(\frac{k_{2}}{\theta}(\mathbf{p}-\mathbf{q})\right) \pi \delta\left(|\mathbf{p}|^{2}-|\mathbf{q}|^{2}\right) \bar{W}(\mathbf{x}, \mathbf{p})-\frac{i k_{2}^{3}}{2 \theta^{4}} f d \mathbf{q} \frac{\Phi_{2}\left(\frac{k_{2}}{\theta}(\mathbf{p}-\mathbf{q})\right)}{|\mathbf{p}|^{2}-|\mathbf{q}|^{2}} \bar{W}(\mathbf{x}, \mathbf{p}) \\
& +\frac{1}{4 \theta^{2}} \int d \mathbf{q} \Phi_{12}(\mathbf{q}) e^{i \tilde{\mathbf{x}} \cdot \mathbf{q}\left(k_{1}^{-1}-k_{2}^{-1}\right)} \pi \delta\left(\frac{\mathbf{q}}{k_{2}} \cdot\left(\mathbf{p}-\frac{\theta \mathbf{q}}{2 k_{1}}\right)\right) \bar{W}\left(\mathbf{x}, \mathbf{p}-\frac{\theta \mathbf{q}}{2 k_{1}}-\frac{\theta \mathbf{q}}{2 k_{2}}\right) \\
& +\frac{1}{4 \theta^{2}} \int d \mathbf{q} \Phi_{12}(\mathbf{q}) e^{i \tilde{\mathbf{x}} \cdot \mathbf{q}\left(k_{1}^{-1}-k_{2}^{-1}\right)} \pi \delta\left(\frac{\mathbf{q}}{k_{1}} \cdot\left(\mathbf{p}-\frac{\theta \mathbf{q}}{2 k_{2}}\right)\right) \bar{W}\left(\mathbf{x}, \mathbf{p}-\frac{\theta \mathbf{q}}{2 k_{1}}-\frac{\theta \mathbf{q}}{2 k_{2}}\right) \\
& +\frac{i}{4 \theta^{2}} \int d \mathbf{q}\left[\frac{1}{\frac{\mathbf{q}}{k_{2}} \cdot\left(\mathbf{p}-\frac{\theta \mathbf{q}}{2 k_{1}}\right)}-\frac{1}{\frac{\mathbf{q}}{k_{1}} \cdot\left(\mathbf{p}-\frac{\theta \mathbf{q}}{2 k_{2}}\right)}\right] \Phi_{12}(\mathbf{q}) e^{i \tilde{\mathbf{x}} \cdot \mathbf{q}\left(k_{1}^{-1}-k_{2}^{-1}\right)} \bar{W}\left(\mathbf{x}, \mathbf{p}-\frac{\theta \mathbf{q}}{2 k_{1}}-\frac{\theta \mathbf{q}}{2 k_{2}}\right)
\end{aligned}
$$

where we have used the fact that in the sense of generalized function

$$
\lim _{\eta \rightarrow 0} \frac{1}{\eta+i \xi}=\pi \delta(\xi)-\frac{i}{\xi}
$$

with the second term giving rise to the Cauchy principal value integral denoted by $f$. From (7) we have the expression for $\langle F\rangle$

$$
\begin{aligned}
\langle F\rangle= & -\frac{i}{2(2 \pi)^{3}} \int e^{-i \mathbf{p} \cdot \mathbf{y}} f_{1}\left(\frac{\mathbf{x}}{k_{1}}+\frac{\mathbf{y}}{2 k_{1}}\right)\left\langle U_{2}^{*}\left(\frac{\mathbf{x}}{k_{2}}-\frac{\mathbf{y}}{2 k_{2}}\right)\right\rangle d \mathbf{y} \\
& +\frac{i}{2(2 \pi)^{3}} \int e^{-i \mathbf{p} \cdot \mathbf{y}}\left\langle U_{1}\left(\frac{\mathbf{x}}{k_{1}}+\frac{\mathbf{y}}{2 k_{1}}\right)\right\rangle f_{2}^{*}\left(\frac{\mathbf{x}}{k_{2}}-\frac{\mathbf{y}}{2 k_{2}}\right) d \mathbf{y} .
\end{aligned}
$$

which depends only on the mean fields $\left\langle U_{1}\right\rangle,\left\langle U_{2}\right\rangle$, both assumed known throughout the paper.

Putting all the terms together with the regularization we arrive at the following MSE

$$
W(\mathbf{x}, \mathbf{p})=\bar{W}(\mathbf{x}, \mathbf{p})+\sqrt{\varepsilon} W_{1}^{\varepsilon}(\mathbf{x}, \tilde{\mathbf{x}}, \mathbf{p})+\varepsilon W_{2}^{\varepsilon}(\mathbf{x}, \tilde{\mathbf{x}}, \mathbf{p})
$$

which satisfies

$$
\begin{aligned}
& \left(\mathbf{p} \cdot \nabla-\frac{1}{\sqrt{\varepsilon}} \mathcal{L}\right) W+i \nu^{\prime} W-F \\
& \quad=\left(i \nu^{\prime}-1\right) \sqrt{\varepsilon} W_{1}^{\varepsilon}+\sqrt{\varepsilon} \mathbf{p} \cdot \nabla_{\mathbf{x}} W_{1}^{\varepsilon}-\sqrt{\varepsilon} \mathcal{L} W_{2}^{\varepsilon}+\left(i \nu^{\prime}-1\right) \varepsilon W_{2}^{\varepsilon}+\varepsilon \mathbf{p} \cdot \nabla_{\mathbf{x}} W_{2}^{\varepsilon} .
\end{aligned}
$$

Unfortunately the right hand side of (24) does not vanish in the strong $L^{2}$-topology but only in the weak topology as in

$$
\lim _{\varepsilon \rightarrow 0} \varepsilon \int d \mathbf{x}\left\langle\left|\int d \mathbf{p} W_{1}^{\varepsilon}\left(\mathbf{x}, \frac{\mathbf{x}}{\varepsilon}, \mathbf{p}\right) \psi(\mathbf{p})\right|^{2}\right\rangle=0, \quad \forall \psi \in L^{2}
$$


(see Appendix B). It is not clear at this point how to justify the preceding argument and construction of asymptotic solution with full mathematical rigor. Fortunately, in the regime of geometrical optics, the rigorous asymptotic result can be obtained by a probabilistic method $^{13}$ and is the same as derived by MSE (see Section 6). Another regime for which the asymptotic result can be fully justified is paraxial waves which we will turn to in the next section.

Due to the assumption (15) and the assumed continuous dependence of the medium fluctuation on the frequency we have $\lim \Phi_{1}=\lim \Phi_{2}=\lim \Phi_{12}=\Phi$. As a consequence, all the Cauchy principal value integrals cancel out. With some changes of variables the governing equation for $\bar{W}$ takes the much simplified form:

$$
\begin{aligned}
\mathbf{p} & \cdot \nabla_{\mathbf{x}} \bar{W}+i \nu^{\prime} \bar{W}-\langle F\rangle \\
& =\frac{\pi k^{3}}{\theta^{4}} \int d \mathbf{q} \Phi\left(\frac{k}{\theta}(\mathbf{p}-\mathbf{q})\right) \delta\left(|\mathbf{p}|^{2}-|\mathbf{q}|^{2}\right)\left[e^{i \mathbf{x} \cdot(\mathbf{p}-\mathbf{q}) \beta} \bar{W}(\mathbf{x}, \mathbf{q})-\bar{W}(\mathbf{x}, \mathbf{p})\right] .
\end{aligned}
$$

The $\delta$-function in the scattering kernel is due to elastic scattering which preserve the wavenumber. When $\beta=0$ (then $\nu_{1}=\nu_{2}$ and $i \nu^{\prime} \sim$ the imaginary part of $\nu$ ), eq. (26) reduce to the standard form of radiative transfer equation for the phase space energy density. ${ }^{5,16,19,22}$ For $\beta>0$, the wave featue is retained in (26). When $\beta \rightarrow \infty$, the first term in

the bracket on the right hand side of (26) drops out, due to rapid phase fluctuation, so the random scattering effect is pure damping:

$$
\mathbf{p} \cdot \nabla_{\mathbf{x}} \bar{W}+i \nu^{\prime} \bar{W}-\langle F\rangle=-\frac{\pi k^{3}}{\theta^{4}} \int d \mathbf{q} \Phi\left(\frac{k}{\theta}(\mathbf{p}-\mathbf{q})\right) \delta\left(|\mathbf{p}|^{2}-|\mathbf{q}|^{2}\right) \bar{W}(\mathbf{x}, \mathbf{p}) .
$$

As a comparison, for Schrödinger equation (10) in the frequency domain, we modify the Wigner distribution as

$$
W(\mathbf{x}, \mathbf{p})=\frac{1}{(2 \pi)^{3}} \int e^{-i \mathbf{p} \cdot \mathbf{y}} \psi_{1}\left(\mathbf{x}+\frac{\varepsilon \hbar \mathbf{y}}{2}\right) \psi_{2}^{*}\left(\mathbf{x}-\frac{\varepsilon \hbar \mathbf{y}}{2}\right) d \mathbf{y}
$$

and in the limit $\varepsilon \rightarrow 0$ obtain the radiative transfer equation following the same procedure

$$
\begin{aligned}
\mathbf{p} & \cdot \nabla_{\mathbf{x}} \bar{W}+i\left(\omega_{2}-\omega_{1}\right) \bar{W}+\frac{2 i}{\hbar} \nu^{\prime} \bar{W}-\langle F\rangle \\
= & \frac{4 \pi}{\hbar^{4}} \int d \mathbf{q} \Phi\left(\frac{\mathbf{p}-\mathbf{q}}{\hbar}\right) \delta\left(|\mathbf{p}|^{2}-|\mathbf{q}|^{2}\right)[\bar{W}(\mathbf{x}, \mathbf{q})-\bar{W}(\mathbf{x}, \mathbf{p})] .
\end{aligned}
$$

The absence of the factor $e^{i \mathbf{x} \cdot(\mathbf{p}-\mathbf{q}) \beta}$ in eq. (27), and therefore the cross-frequency interference, is the main characteristic of $2 \mathrm{f}-\mathrm{RT}$ for quantum waves.

\section{Paraxial 2f-RT: anisotropic medium}

Forward-scattering approximation, also called paraxial approximation, is valid when backscattering is negligible and, as we show now, this is the case for anisotropic media fluctuating 
slowly in the (longitudinal) direction of propagation. Let $z$ denote the longitudinal coordinate and $\mathbf{x}_{\perp}$ the transverse coordinates. Let $p$ and $\mathbf{p}_{\perp}$ denote the longitudinal and transverse components of $\mathbf{p} \in \mathbb{R}^{3}$, respectively. Let $\mathbf{q}=\left(q, \mathbf{q}_{\perp}\right) \in \mathbb{R}^{3}$ be likewise defined.

Consider now a highly anisotropic spectral density for a medium fluctuating much more slowly in the longitudinal direction, i.e. replacing $\Phi((\mathbf{p}-\mathbf{q}) k / \theta)$ in (26) by

$$
\frac{1}{\eta} \Phi\left(\frac{k}{\eta \theta}(p-q), \frac{k}{\theta}\left(\mathbf{p}_{\perp}-\mathbf{q}_{\perp}\right)\right), \quad \eta \ll 1,
$$

which, in the limit $\eta \rightarrow 0$, tends to

$$
\frac{\theta}{k} \delta(p-q) \int d w \Phi\left(w, \frac{k}{\theta}\left(\mathbf{p}_{\perp}-\mathbf{q}_{\perp}\right)\right) .
$$

Writing $\bar{W}=\bar{W}\left(z, \mathbf{x}_{\perp}, p, \mathbf{p}_{\perp}\right)$ we can approximate eq. (26) by

$$
\begin{aligned}
p \partial_{z} \bar{W} & +\mathbf{p}_{\perp} \cdot \nabla_{\mathbf{x}_{\perp}} \bar{W}+i \nu^{\prime} \bar{W}-\langle F\rangle \\
= & \frac{\pi k^{2}}{\theta^{3}} \int d \mathbf{q}_{\perp} \int d w \Phi\left(w, \frac{k}{\theta}\left(\mathbf{p}_{\perp}-\mathbf{q}_{\perp}\right)\right) \delta\left(\left|\mathbf{p}_{\perp}\right|^{2}-\left|\mathbf{q}_{\perp}\right|^{2}\right) \\
& \times\left[e^{i \mathbf{x}_{\perp} \cdot\left(\mathbf{p}_{\perp}-\mathbf{q}_{\perp}\right) \beta} \bar{W}\left(z, \mathbf{x}_{\perp}, p, \mathbf{q}_{\perp}\right)-\bar{W}\left(z, \mathbf{x}_{\perp}, p, \mathbf{p}_{\perp}\right)\right]
\end{aligned}
$$

Eq. (29) is identical to the 2f-RT equation rigorously derived directly from the paraxial wave equation for similar anisotropic media. ${ }^{11,12}$ This is somewhat surprising in view of the

different scaling factors in the definition of two-frequency Wigner distributions in the two cases.

Note that in eq. (29) the longitudinal momentum $p$ plays the role of a parameter and does not change during propagation and scattering. An important implication of this observation is that eq. (29) can be solved as an evolution equation in the direction of increasing $z$ with the one-sided boundary condition (e.g. at $z=$ const.). In other words, the influence from the other boundary vanishes as the longitudinal direction is infinitely long. The initial value problem of (29) is much easier to solve than the boundary value problem of (26) $)$.

\section{Two-frequency geometrical radiative transfer (2f-GRT)}

Let us consider the further limit $\theta \ll 1$ when the wavelength is much shorter than the correlation length of the medium fluctuation. To this end, the following form is more convenient to work with

$$
\begin{aligned}
\mathbf{p} & \cdot \nabla_{\mathbf{x}} \bar{W}+i \nu^{\prime} \bar{W}-\langle F\rangle \\
& =\frac{\pi k}{2 \theta^{2}} \int d \mathbf{q} \Phi(\mathbf{q}) \delta\left(\mathbf{q} \cdot\left(\mathbf{p}-\frac{\theta \mathbf{q}}{2 k}\right)\right)\left[e^{i \mathbf{x} \cdot \mathbf{q} \beta \theta / k} \bar{W}\left(\mathbf{x}, \mathbf{p}-\frac{\theta \mathbf{q}}{k}\right)-\bar{W}(\mathbf{x}, \mathbf{p})\right]
\end{aligned}
$$


which is obtained from eq. (26) after a change of variables. We expand the right hand side of (30) in $\theta$ and pass to the limit $\theta \rightarrow 0$ to obtain

$$
\mathbf{p} \cdot \nabla_{\mathbf{x}} \bar{W}+i \nu^{\prime} \bar{W}-\langle F\rangle=\frac{1}{4 k}\left(\nabla_{\mathbf{p}}-i \beta \mathbf{x}\right) \cdot \mathbf{D} \cdot\left(\nabla_{\mathbf{p}}-i \beta \mathbf{x}\right) \bar{W}
$$

with the (momentum) diffusion coefficient

$$
\mathbf{D}(\mathbf{p})=\pi \int \Phi(\mathbf{q}) \delta(\mathbf{p} \cdot \mathbf{q}) \mathbf{q} \otimes \mathbf{q} d \mathbf{q} .
$$

The symmetry $\Phi(\mathbf{p})=\Phi(-\mathbf{p})$ plays an explicit role here in rendering the right hand side of eq. (30) a second-order operator in the limit $\theta \rightarrow 0$. Eq. (31) can be rigorously derived from geometrical optics by a probabilistic method. ${ }^{13}$

\section{A. Spatial (frequency) spread and coherence bandwidth}

Through dimensional analysis, eq. (31) yields qualitative information about important physical parameters of the stochastic medium. To show this, let us assume for simplicity the isotropy of the medium, i.e. $\Phi(\mathbf{p})=\Phi(|\mathbf{p}|)$, so that $\mathbf{D}=C|\mathbf{p}|^{-1} \Pi(\mathbf{p})$ where

$$
C=\frac{\pi}{3} \int \delta\left(\frac{\mathbf{p}}{|\mathbf{p}|} \cdot \frac{\mathbf{q}}{|\mathbf{q}|}\right) \Phi(|\mathbf{q}|)|\mathbf{q}| d \mathbf{q}
$$

is a constant and $\Pi(\mathbf{p})$ the orthogonal projection onto the plane perpendicular to $\mathbf{p}$. In view of (31) $C$ (and $\mathbf{D}$ ) has the dimension of inverse length while the variables $\mathbf{x}$ and $\mathbf{p}$ are dimensionless.

Now consider the following change of variables

$$
\mathbf{x}=\sigma_{x} k \tilde{\mathbf{x}}, \quad \mathbf{p}=\sigma_{p} \tilde{\mathbf{p}} / k, \quad \beta=\beta_{c} \tilde{\beta}
$$

where $\sigma_{x}$ and $\sigma_{p}$ are respectively the spreads in position and spatial frequency, and $\beta_{c}$ is the coherence bandwidth. Let us substitute (34) into eq. (31) and aim for the standard form

$$
\tilde{\mathbf{p}} \cdot \nabla_{\tilde{\mathbf{x}}} \bar{W}+i \nu^{\prime} \bar{W}-\langle F\rangle=\left(\nabla_{\tilde{\mathbf{p}}}-i \tilde{\beta} \tilde{\mathbf{x}}\right) \cdot|\tilde{\mathbf{p}}|^{-1} \Pi(\tilde{\mathbf{p}})\left(\nabla_{\tilde{\mathbf{p}}}-i \tilde{\beta} \tilde{\mathbf{x}}\right) \bar{W} .
$$

The 1-st term on the left side yields the first duality relation

$$
\sigma_{x} / \sigma_{p} \sim 1 / k^{2}
$$

The balance of terms in each pair of parentheses yields the second duality relation

$$
\sigma_{x} \sigma_{p} \sim \frac{1}{\beta_{c}}
$$

whose left hand side is the space-spread-bandwidth product. Finally the removal of the constant $C$ determines

$$
\sigma_{p} \sim k^{2 / 3} C^{1 / 3}
$$


from which $\sigma_{x}$ and $\beta_{c}$ can be determined by using (36) and (37):

$$
\sigma_{x} \sim k^{-4 / 3} C^{1 / 3}, \quad \beta_{c} \sim k^{2 / 3} C^{-2 / 3}
$$

We do not know if, as it stands, eq. (35) is analytically solvable but we can solve analytically for its boundary layer behavior.

\section{B. Boundary layer asymptotics: paraxial 2f-GRT}

Consider the half space $z \geq 0$ occupied by the random medium and a collimated narrowband beam propagating in the $z$ direction and incident normal to the boundary $(z=0)$ of the medium. Near the point of incidence on the boundary the corresponding two-frequency Wigner distribution would be highly concentrated at the longitudinal momentum, say, $p=1$. Hence we can assume that the projection $\Pi(\mathbf{p})$ in (35) is effectively just the projection onto the transverse plane coordinated by $\mathbf{x}_{\perp}$ and approximate eq. (31) by

$$
\left[\partial_{z}+\mathbf{p}_{\perp} \cdot \nabla_{\mathbf{x}_{\perp}}\right] \bar{W}+i \nu^{\prime} \bar{W}-\langle F\rangle=\frac{C_{\perp}}{4 k|p|}\left(\nabla_{\mathbf{p}_{\perp}}-i \beta \mathbf{x}_{\perp}\right)^{2} \bar{W}
$$

where the constant $C_{\perp}$ is the paraxial approximation of (32) for $|p|=1$ :

$$
C_{\perp}=\frac{\pi}{2} \int \Phi\left(0, \mathbf{q}_{\perp}\right)\left|\mathbf{q}_{\perp}\right|^{2} d \mathbf{q}_{\perp} .
$$

Here we have assumed the isotropy of $\Phi$ in the transverse dimensions. Note that the longitudinal (momentum) diffusion vanishes and that the longitudinal momentum $p$ plays the role of a parameter in eq. (39) which then can be solved in the direction of increasing $z$ as an

evolution equation with initial data given at a fixed $z$. This is another instance of paraxial approximation.

Let $\sigma_{*}$ be the spatial spread in the transverse coordinates $\mathbf{x}_{\perp}, \ell_{c}$ the coherence length in the transverse dimensions and $\beta_{c}$ the coherence bandwidth. Let $L$ be the scale of the boundary layer. We then seek the following change of variables

$$
\tilde{\mathbf{x}}_{\perp}=\frac{\mathbf{x}_{\perp}}{\sigma_{*} k}, \quad \tilde{\mathbf{p}}_{\perp}=\mathbf{p}_{\perp} k \ell_{c}, \quad \tilde{z}=\frac{z}{L k}, \quad \tilde{\beta}=\frac{\beta}{\beta_{c}}
$$

to remove all the physical parameters from (39) and to aim for the form

$$
\partial_{\tilde{z}} \bar{W}+\tilde{\mathbf{p}}_{\perp} \cdot \nabla_{\tilde{\mathbf{x}}_{\perp}} \bar{W}+L k i \nu^{\prime} \bar{W}-L k\langle F\rangle=\left(\nabla_{\tilde{\mathbf{p}}_{\perp}}-i \tilde{\beta} \tilde{\mathbf{x}}_{\perp}\right)^{2} \bar{W}
$$

The same reasoning as above now leads to

$$
\ell_{c} \sigma_{*} \sim L / k, \quad \sigma_{*} / \ell_{c} \sim 1 / \beta_{c}, \quad \ell_{c} \sim k^{-1} L^{-1 / 2} C_{\perp}^{-1 / 2}
$$

and hence

$$
\sigma_{*} \sim L^{3 / 2} C_{\perp}^{1 / 2}, \quad \beta_{c} \sim k^{-1} C_{\perp}^{-1} L^{-2} .
$$


The layer thickness $L$ may be determined by $\ell_{c} \sim 1$, i.e. $L \sim k^{-2} C_{\perp}^{-1}$.

After the inverse Fourier transform eq. (41) becomes

$$
\partial_{\tilde{z}} \Gamma-i \nabla_{\tilde{\mathbf{y}}_{\perp}} \cdot \nabla_{\tilde{\mathbf{x}}_{\perp}} \Gamma+L k i \nu^{\prime} \Gamma-L k\langle F\rangle=-\left|\tilde{\mathbf{y}}_{\perp}+\tilde{\beta}_{\tilde{\mathbf{x}}_{\perp}}\right|^{2} \Gamma
$$

which is the governing equation for the two-frequency mutual coherence in the normalized variables. With data given on $\tilde{z}=0$ and vanishing far-field boundary condition in the transverse directions, Eq. (42) can be solved analytically and its Green function is given by

$$
\begin{aligned}
& \frac{e^{-i L k \nu^{\prime}}(i 4 \tilde{\beta})^{1 / 2}}{(2 \pi)^{2} \tilde{z} \sinh \left[(i 4 \tilde{\beta})^{1 / 2} \tilde{z}\right]} \exp \left[\frac{1}{i 4 \tilde{\beta} \tilde{z}}\left|\tilde{\mathbf{y}}_{\perp}-\tilde{\beta} \tilde{\mathbf{x}}_{\perp}-\mathbf{y}_{\perp}^{\prime}+\tilde{\beta} \mathbf{x}_{\perp}^{\prime}\right|^{2}\right] \\
& \times \exp \left[-\frac{\operatorname{coth}\left[(i 4 \tilde{\beta})^{1 / 2} \tilde{z}\right]}{(i 4 \tilde{\beta})^{1 / 2}}\left|\tilde{\mathbf{y}}_{\perp}+\tilde{\beta} \tilde{\mathbf{x}}_{\perp}-\frac{\mathbf{y}_{\perp}^{\prime}+\tilde{\beta} \mathbf{x}_{\perp}^{\prime}}{\cosh \left[(i 4 \tilde{\beta})^{1 / 2} \tilde{z}\right]}\right|^{2}\right] \\
& \times \exp \left[-\frac{\tanh \left[(i 4 \tilde{\beta})^{1 / 2} \tilde{z}\right]}{(i 4 \tilde{\beta})^{1 / 2}}\left|\mathbf{y}_{\perp}^{\prime}+\tilde{\beta} \mathbf{x}_{\perp}^{\prime}\right|^{2}\right] .
\end{aligned}
$$

Formula (43) is consistent with the asymptotic result in the literature which mainly concerns with the cross-frequency correlation of intensity. In the radiative transfer regime considered here, the cross-spectral correlation of intensity is the square of the two-frequency mutual coherence and has the commonly accepted form ${ }^{15,24,26}$

$$
\exp [-2 \sqrt{2 \tilde{\beta}}]
$$

which is just the large $\tilde{\beta}$ asymptotic of the squared factor $\left|\sinh \left[(i 4 \tilde{\beta})^{1 / 2} \tilde{z}\right]\right|^{-2}$ in (43) at $\tilde{z}=1$ ( see $^{13}$ for detailed comparison). Moreover (43) provides detailed information about the simultaneous dependence of the mutual coherence on the frequency difference and spatial displacement for $\tilde{z} \in(0,1) \cdot{ }^{23,26}$

Surprisingly, a closely related equation arises in the two-frequency formulation of the Markovian approximation of the paraxial waves. ${ }^{8}$ The closed form solution is crucial for analyzing the performance of time reversal communication with broadband signals. ${ }^{10}$ The solution procedure for (43) is similar to that given elsewhere ${ }^{10}$ and is omitted here.

\section{C. Paraxial 2f-GRT in anisotropic media}

We use here the setting and notation defined in Section 5 for anisotropic media. For simplicity we will set $p=1$ and omit writing it out in $\bar{W}$. In view of (28) we replace $\Phi(\mathbf{q})$ in (32) by

$$
\delta(q) \int d w \Phi\left(w, \mathbf{q}_{\perp}\right)
$$

and obtain the transverse diffusion coefficient

$$
\mathbf{D}_{\perp}\left(\mathbf{p}_{\perp}\right)=\pi \int d \mathbf{q}_{\perp} \int d w \Phi\left(w, \mathbf{q}_{\perp}\right) \delta\left(\mathbf{p}_{\perp} \cdot \mathbf{q}_{\perp}\right) \mathbf{q}_{\perp} \otimes \mathbf{q}_{\perp}
$$


whereas the longitudinal diffusion coefficient is zero.

For simplicity we assume the isotropy in the transverse dimensions, $\Phi\left(w, \mathbf{p}_{\perp}\right)=\Phi\left(w,\left|\mathbf{p}_{\perp}\right|\right)$, so that $\mathbf{D}_{\perp}=C_{\perp}\left|\mathbf{p}_{\perp}\right|^{-1} \Pi_{\perp}\left(\mathbf{p}_{\perp}\right)$ where

$$
C_{\perp}=\frac{\pi}{2} \int \delta\left(\frac{\mathbf{p}_{\perp}}{\left|\mathbf{p}_{\perp}\right|} \cdot \frac{\mathbf{q}_{\perp}}{\left|\mathbf{q}_{\perp}\right|}\right) \Phi\left(w,\left|\mathbf{q}_{\perp}\right|\right)\left|\mathbf{q}_{\perp}\right| d w d \mathbf{q}_{\perp}
$$

is a constant and $\Pi_{\perp}\left(\mathbf{p}_{\perp}\right)$ is the orthogonal projection onto the transverse line perpendicular to $\mathbf{p}_{\perp}$. Hence eq. (31) reduces to

$$
\begin{aligned}
& {\left[\partial_{z}+\mathbf{p}_{\perp} \cdot \nabla_{\mathbf{x}_{\perp}}\right] \bar{W}+i \nu^{\prime} \bar{W}-\langle F\rangle} \\
& \quad=\frac{C_{\perp}}{4 k}\left(\nabla_{\mathbf{p}_{\perp}}-i \beta \mathbf{x}_{\perp}\right) \cdot\left|\mathbf{p}_{\perp}\right|^{-1} \Pi_{\perp}\left(\mathbf{p}_{\perp}\right)\left(\nabla_{\mathbf{p}_{\perp}}-i \beta \mathbf{x}_{\perp}\right) \bar{W} .
\end{aligned}
$$

Alternatively, eq. (45) can also be derived from eq. (29) by taking the geometrical optics limit as described in the beginning of Section 6 .

Consider the change of variables (40) to remove all the physical parameters from (45) and to aim for the form

$$
\begin{aligned}
& {\left[\partial_{\tilde{z}}+\tilde{\mathbf{p}}_{\perp} \cdot \nabla_{\tilde{\mathbf{x}}_{\perp}}\right] \bar{W}+L k i \nu^{\prime} \bar{W}-L k\langle F\rangle} \\
& \quad=\left(\nabla_{\tilde{\mathbf{p}}_{\perp}}-i \tilde{\beta} \tilde{\mathbf{x}}_{\perp}\right) \cdot\left|\tilde{\mathbf{p}}_{\perp}\right|^{-1} \Pi_{\perp}\left(\tilde{\mathbf{p}}_{\perp}\right)\left(\nabla_{\tilde{\mathbf{p}}_{\perp}}-i \tilde{\beta} \tilde{\mathbf{x}}_{\perp}\right) \bar{W}
\end{aligned}
$$

where $L$ should be interpreted as the distance of propagation.

Following the same line of reasoning, we obtain that

$$
\ell_{c} \sigma_{*} \sim L / k, \quad \sigma_{*} / \ell_{c} \sim 1 / \beta_{c}, \quad \ell_{c} \sim C_{\perp}^{-1 / 3} L^{-1 / 3} k^{-1}
$$

and hence

$$
\sigma_{*} \sim C_{\perp}^{1 / 3} L^{4 / 3}, \quad \beta_{c} \sim C_{\perp}^{-2 / 3} L^{-5 / 3} k^{-1}
$$

Unlike (39) it is unclear if a closed-form solution to eq. (45) exists or not.

\section{Discussion and conclusion}

The standard (one-frequency) RT can be formally derived from the wave equation in at least two ways: the diagrammatic expansion method, as the ladder approximation of the Bethe-Salpeter equation, ${ }^{19,26}$ and the multi-scale expansion method advocated here. ${ }^{1}$ The latter is considerably simpler than the former in terms of the amount of calculation involved. Both approaches have been developed with full mathematical rigor in some special cases ( see $^{7,9}$ and the references therein). There are two regimes for which the 2f-RT equation has been derived with full mathematical rigor: first, for the paraxial wave equation by using the so called martingale method in probability theory; ${ }^{11,12}$ second, for the spherical waves in 
geometrical optics by the path-integration method. ${ }^{13}$ These rigorous results coincide with those derived here for the respective regimes and hence support the validity of MSE.

Within the framework of 2f-RT, a paraxial form arises naturally in anisotropic media which fluctuate slowly in the longitudinal direction. Another form of paraxial 2f-RT takes place in the boundary layer asymptotics of isotropic media. The latter equation turns out to be exactly solvable and the boundary layer behavior is given in a closed form, revealing highly non-trivial structure of the two-frequency mutual coherence. In any case, dimensional analysis with the 2f-GRT equations yields qualitative scaling behavior of the spatial spread, the spatial frequency spread and the coherent bandwidth in various regimes.

From the point of view of computation, especially Monte Carlo simulation, it appears to be natural to introduce the new quantity

$$
\mathfrak{W}(\mathbf{x}, \mathbf{p})=e^{-i \beta \mathbf{x} \cdot \mathbf{p}} \bar{W}(\mathbf{x}, \mathbf{p})
$$

and rewrite eq. (26) in the following form

$$
\begin{aligned}
\mathbf{p} & \cdot \nabla_{\mathbf{x}} \mathfrak{W}+i \beta|\mathbf{p}|^{2} \mathfrak{W}+i \nu^{\prime} \mathfrak{W}-e^{-i \beta \mathbf{x} \cdot \mathbf{p}}\langle F\rangle \\
= & \frac{\pi k^{3}}{\theta^{4}} \int d \mathbf{q} \Phi\left(\frac{k}{\theta}(\mathbf{p}-\mathbf{q})\right) \delta\left(|\mathbf{p}|^{2}-|\mathbf{q}|^{2}\right)[\mathfrak{W}(\mathbf{x}, \mathbf{q})-\mathfrak{W}(\mathbf{x}, \mathbf{p})] .
\end{aligned}
$$

The solution $\mathfrak{W}$ can then be expressed as a path integration over the Markov process generated by the operator $\mathcal{A}$ defined by

$$
\mathcal{A} \mathfrak{W}=-\mathbf{p} \cdot \nabla_{\mathrm{x}} \mathfrak{W}+\frac{\pi k^{3}}{\theta^{4}} \int d \mathbf{q} \Phi\left(\frac{k}{\theta}(\mathbf{p}-\mathbf{q})\right) \delta\left(|\mathbf{p}|^{2}-|\mathbf{q}|^{2}\right)[\mathfrak{W}(\mathbf{x}, \mathbf{q})-\mathfrak{W}(\mathbf{x}, \mathbf{p})]
$$

when $V$ is real-valued and $\Phi$ is nonnegative. We will pursue this observation in a separate publication. ${ }^{13}$

\section{References}

1. Bensoussan, A. \& Lions, J. L. \& Papanicolaou, G. C., Asymptotic Analysis for Periodic Structures (North-Holland, Amsterdam, 1978).

2. R. Berkovits and S. Feng, "Correlations in coherent multiple scattering," Phys. Rep. 238, 135-172 (1994).

3. M. Born and W. Wolf, Principles of Optics, 7-th (expanded) edition (Cambridge University Press, 1999).

4. A. Bronshtein and I.T. Lu and R. Mazar, "Reference-wave solution for the two-frequency propagator in a statistically homogeneous random medium," Phys. Rev. E. 69, 016607 (2004).

5. S. Chandrasekhar, Radiative Transfer (Dover Publications, New York, 1960). 
6. D. Dragoman, "The Wigner distribution function in optics and optoelectronics," in Progress in Optics, E. Wolf, ed. (Elsevier, 1997) Vol. 37, pp. 1-56.

7. A. C. Fannjiang, "Self-averaging scaling limits for random parabolic waves, " Arch. Rat. Mech. Anal. 175:3, 343 - 387(2005).

8. A. C. Fannjiang, "White-noise and geometrical optics limits of Wigner-Moyal equation for wave beams in turbulent media II. Two-frequency Wigner distribution formulation, " J. Stat. Phys. 120, 543-586 (2005).

9. A. C. Fannjiang, "Self-averaging radiative transfer for parabolic waves, " Compt. Rend. Math. 342, 109-114 (2006).

10. A. C. Fannjiang, "Information transfer in disordered media by broadband time reversal: stability, resolution and capacity," Nonlinearity 19, 2425-2439 (2006).

11. A. C. Fannjiang, "Radiative transfer limit of two-frequency Wigner distribution for random parabolic waves: An exact solution," Compt. Rend. Phys. 8, 267-271 (2007).

12. A. C. Fannjiang, "Self-averaging scaling limits of two-frequency Wigner distribution for random paraxial waves, " J. Phys. A.: Math. Theor. 40, 5025-5044 (2007).

13. A. C. Fannjiang, "Space-frequency correlation of classical waves in disordered media: high-frequency asymptotics, " to appear.

14. G.W. Forbes, V.I. Man'ko, H.M. Ozaktas, R. Simon, K.B. Wolf eds., Wigner Distributions and Phase Space in Optics, 2000 (feature issue, J. Opt. Soc. Am. A 17 No. 12).

15. A. Z. Genack, "Optical transmission in disordered media," Phys. Rev. Lett. 58, 20432046 (1987).

16. E. Hopf, Mathematical Problems of Radiative Equilibrium (Cambridge University Press, Cambridge, 1934).

17. A. Ishimaru, Wave Propagation and Scattering in Random Media, Vol. I \& II (Academic, New York, 1978).

18. L. Mandel and E. Wolf, Optical Coherence and Quantum Optics (Cambridge University Press, 1995).

19. M. Mishchenko, L. Travis, A. Lacis, Multiple Scattering of Light by Particles: Radiative Transfer and Coherent Backscattering (Cambridge University Press, Cambridge, 2006).

20. L. Ryzhik, G. Papanicolaou and J.B. Keller, "Transport equations for elastic and other waves in random media," Wave Motion 24, 327-370 (1996).

21. G. Samelsohn and V. Freilikher, "Two-frequency mutual coherence function and pulse propagation in random media," Phys. Rev. E. 65, 046617 (2002).

22. A. Schuster, "Radiation through a foggy atmosphere," Astrophys. J. 21, 1-22 (1905).

23. P. Sebbah, B. Hu, A.Z. Genack, R. Pnini and B. Shapiro, "Spatial-field correlation: the building block of mesoscopic fluctuations," Phys. Rev. Lett. 88, 123901 (2002).

24. B. Shapiro, "Large intensity fluctuations for wave propagation in random media," Phys. 
Rev. Lett. 57, 2168-2171 (1986)

25. H. Spohn, "Kinetic equations from Hamiltonian dynamics: Markovian limits, " Rev. Mod. Phys. 53, 569-615 (1980).

26. M.C.W. van Rossum and Th. M. Nieuwenhuizen, "Multiple scattering of classical waves: microscopy, mesoscopy, and diffusion," Rev. Mod. Phys. 71, 313-371 (1999).

\section{A. Derivation of eq. (6)}

Applying the operator $\mathbf{p} \cdot \nabla$ to the definition (3) we obtain

$$
\begin{aligned}
\mathbf{p} \cdot \nabla_{\mathbf{x}} W= & \frac{1}{(2 \pi)^{3}} \int e^{-i \mathbf{p} \cdot \mathbf{y}} 2 \mathbf{p} \cdot \nabla_{\mathbf{y}} U_{1}\left(\frac{\mathbf{x}}{k_{1}}+\frac{\mathbf{y}}{2 k_{1}}\right) U_{2}^{*}\left(\frac{\mathbf{x}}{k_{2}}-\frac{\mathbf{y}}{2 k_{2}}\right) d \mathbf{y} \\
& -\frac{1}{(2 \pi)^{3}} \int e^{-i \mathbf{p} \cdot \mathbf{y}} U_{1}\left(\frac{\mathbf{x}}{k_{1}}+\frac{\mathbf{y}}{2 k_{1}}\right) 2 \mathbf{p} \cdot \nabla_{\mathbf{y}} U_{2}^{*}\left(\frac{\mathbf{x}}{k_{2}}-\frac{\mathbf{y}}{2 k_{2}}\right) d \mathbf{y} \\
= & \frac{2 i}{(2 \pi)^{3}} \int\left(\nabla_{\mathbf{y}} e^{-i \mathbf{p} \cdot \mathbf{y}}\right) \cdot \nabla_{\mathbf{y}} U_{1}\left(\frac{\mathbf{x}}{k_{1}}+\frac{\mathbf{y}}{2 k_{1}}\right) U_{2}^{*}\left(\frac{\mathbf{x}}{k_{2}}-\frac{\mathbf{y}}{2 k_{2}}\right) d \mathbf{y} \\
& -\frac{2 i}{(2 \pi)^{3}} \int\left(\nabla_{\mathbf{y}} e^{-i \mathbf{p} \cdot \mathbf{y}}\right) U_{1}\left(\frac{\mathbf{x}}{k_{1}}+\frac{\mathbf{y}}{2 k_{1}}\right) \cdot \nabla_{\mathbf{y}} U_{2}^{*}\left(\frac{\mathbf{x}}{k_{2}}-\frac{\mathbf{y}}{2 k_{2}}\right) d \mathbf{y} .
\end{aligned}
$$

Integrating by parts with the first $\nabla_{\mathbf{y}}$ in the above integrals, we have

$$
\begin{aligned}
\mathbf{p} \cdot \nabla_{\mathbf{x}} W= & -\frac{2 i}{(2 \pi)^{3}} \int e^{-i \mathbf{p} \cdot \mathbf{y}} \nabla_{\mathbf{y}}^{2} U_{1}\left(\frac{\mathbf{x}}{k_{1}}+\frac{\mathbf{y}}{2 k_{1}}\right) U_{2}^{*}\left(\frac{\mathbf{x}}{k_{2}}-\frac{\mathbf{y}}{2 k_{2}}\right) d \mathbf{y} \\
& +\frac{2 i}{(2 \pi)^{3}} \int e^{-i \mathbf{p} \cdot \mathbf{y}} U_{1}\left(\frac{\mathbf{x}}{k_{1}}+\frac{\mathbf{y}}{2 k_{1}}\right) \nabla_{\mathbf{y}}^{2} U_{2}^{*}\left(\frac{\mathbf{x}}{k_{2}}-\frac{\mathbf{y}}{2 k_{2}}\right) d \mathbf{y}
\end{aligned}
$$

where the other resulting terms are canceled with each other. From eq. (21),

$$
\nabla_{\mathbf{y}}^{2} U_{j}\left(\frac{\mathbf{x}}{k_{j}}+\frac{\mathbf{y}}{2 k_{j}}\right)=-\frac{1}{4}\left(\nu_{j}+V_{j}\left(\frac{\mathbf{x}}{k_{j}}+\frac{\mathbf{y}}{2 k_{j}}\right)\right) U_{j}\left(\frac{\mathbf{x}}{k_{j}}+\frac{\mathbf{y}}{2 k_{j}}\right)+\frac{1}{4} f_{j}\left(\frac{\mathbf{x}}{k_{j}}+\frac{\mathbf{y}}{2 k_{j}}\right) .
$$

Using (48) in (47) we arrive at eq. (6).

\section{B. Weak convergence of corrector}

First we show that the corrector does not vanish in the in the mean-square norm in any dimension, i.e. $\lim _{\varepsilon \rightarrow 0} \varepsilon \int\left\langle\left|W_{1}^{\varepsilon}\right|^{2}\right\rangle d \mathbf{x} d \mathbf{p}>0$ in general. For simplicity, consider only the term involving, say, $\hat{V}_{1}$ in the expression (21). A straightforward calculation shows

$$
\begin{aligned}
& \lim _{\varepsilon \rightarrow 0} \frac{1}{4 \theta^{2}} \int d \mathbf{p} d \mathbf{x} d \mathbf{q} \Phi_{1}(\mathbf{q}) \frac{\varepsilon}{\varepsilon^{2}+\left(\mathbf{p} \cdot \mathbf{q} / k_{1}\right)^{2}}\left|\bar{W}\left(\mathbf{x}, \mathbf{p}-\frac{\theta \mathbf{q}}{2 k_{1}}\right)\right|^{2} \\
& \quad=\frac{k \pi}{4 \theta^{2}} \int d \mathbf{p} d \mathbf{x} d \mathbf{q} \Phi(\mathbf{q}) \delta(\mathbf{p} \cdot \mathbf{q})\left|\bar{W}\left(\mathbf{x}, \mathbf{p}-\frac{\theta \mathbf{q}}{2 k}\right)\right|^{2}
\end{aligned}
$$

which is positive in general. 
Next we show that the corrector vanishes in the weak topology

$$
\lim _{\varepsilon \rightarrow 0} \varepsilon \int d \mathbf{x}\left\langle\left|\int d \mathbf{p} W_{1}^{\varepsilon}\left(\mathbf{x}, \frac{\mathbf{x}}{\varepsilon}, \mathbf{p}\right) \psi(\mathbf{p})\right|^{2}\right\rangle=0, \quad \forall \psi \in L^{2} .
$$

It suffices to prove (49) for any smooth, compactly supported function $\psi$. For the term involving $\hat{V}_{1}$ only, we have

$$
\begin{aligned}
& \lim _{\varepsilon \rightarrow 0} \frac{\varepsilon}{4 \theta^{2}} \int d \mathbf{p} d \mathbf{p}^{\prime} d \mathbf{x} d \mathbf{q} \frac{\Phi_{1}(\mathbf{q}) \psi(\mathbf{p}) \psi^{*}\left(\mathbf{p}^{\prime}\right)}{\left(\varepsilon+i \mathbf{p} \cdot \mathbf{q} / k_{1}\right)\left(\varepsilon-i \mathbf{p}^{\prime} \cdot \mathbf{q} / k_{1}\right)} \bar{W}\left(\mathbf{x}, \mathbf{p}-\frac{\theta \mathbf{q}}{2 k_{1}}\right) \bar{W}^{*}\left(\mathbf{x}, \mathbf{p}^{\prime}-\frac{\theta \mathbf{q}}{2 k_{1}}\right) \\
= & \lim _{\varepsilon \rightarrow 0} \frac{\varepsilon k_{1}^{2}}{4 \theta^{2}} \int d \mathbf{q} \frac{\Phi_{1}(\mathbf{q})}{|\mathbf{q}|^{2}}\left[\pi \int d \mathbf{p} \delta(\mathbf{p} \cdot \hat{\mathbf{q}}) \psi(\mathbf{p}) \bar{W}\left(\mathbf{x}, \mathbf{p}-\frac{\theta \mathbf{q}}{2 k_{1}}\right)-f d \mathbf{p} \frac{i \psi(\mathbf{p})}{\mathbf{p} \cdot \hat{\mathbf{q}}} \bar{W}\left(\mathbf{x}, \mathbf{p}-\frac{\theta \mathbf{q}}{2 k_{1}}\right)\right] \\
& \times\left[\pi \int d \mathbf{p}^{\prime} \delta\left(\mathbf{p}^{\prime} \cdot \hat{\mathbf{q}}\right) \psi^{*}\left(\mathbf{p}^{\prime}\right) \bar{W}^{*}\left(\mathbf{x}, \mathbf{p}^{\prime}-\frac{\theta \mathbf{q}}{2 k_{1}}\right)+f d \mathbf{p}^{\prime} \frac{i \psi^{*}\left(\mathbf{p}^{\prime}\right)}{\mathbf{p}^{\prime} \cdot \hat{\mathbf{q}}} \bar{W}^{*}\left(\mathbf{x}, \mathbf{p}^{\prime}-\frac{\theta \mathbf{q}}{2 k_{1}}\right)\right]
\end{aligned}
$$

where $\hat{\mathbf{q}}=\mathbf{q} /|\mathbf{q}|$ for sufficiently smooth $\bar{W}, \Phi$ and rapidly decaying $\Phi$. The essential point now is that $|\mathbf{q}|^{-2}$ is an integrable singularity in three dimensions and hence the above expression vanishes in the limit. 